\title{
Reliability of mRNA Profiling: Verification for Samples with Different Complexities
}

BioTechniques 30:1352-1357 (June 2001)

\begin{abstract}
Bérengère Pradet-Balade, Florence Boulmé1, Ernst W. Müllner $^{1}$, and Jose A. Garcia-Sanz Department of Immunology and Oncology, Centro Nacional de Biotecnologia, Madrid, Spain, and ${ }^{1}$ Institute of Medical Biochemistry, Vienna Biocenter, Vienna, Austria
\end{abstract}

\section{INTRODUCTION}

mRNA profiling has become, within a relatively short time period, an invaluable tool for the simultaneous analysis of mRNA expression levels for up to thousands of genes upon physiological, developmental, and pathological processes, as well as in responses to drug treatment $(7,11,15,16,20,22,30,33-35)$. Indeed, new applications and experimental procedures using cDNA arrays are being reported in rapid sequence $(9,18,25,31,36,38)$. Crucial for accurate analysis is the issue of data normalization, which remains to be solved in a satisfactory manner.

In yeast, normalization is usually assessed by hybridization of the target to yeast genomic DNA (8). In higher eukaryotes, this obvious control cannot be applied because, unlike in yeast, only a small part of the genome is expressed (10). Normalization has been performed by using a small subset of housekeeping genes, whose expression levels are presumed to remain unchanged $(1,13)$, or by the mean filter hybridization signal (38). Expression of housekeeping genes is regulated in many circumstances [e.g., the transition from nonproliferating to proliferating cells $(3,21,25,39)]$. Theoretically, reliable normalization would require one to establish experimentally, for each system, a subset of constantly expressed genes (5). Furthermore, normalization using the average filter hybridization signal is only reliable for closely related samples, in which expression levels for the majority of genes remain unchanged (5). For divergent samples, however, this normalization will also be unreliable because the fraction of genes showing altered transcription rates increases (5) [e.g., during $\mathrm{T}$ lymphocyte activation, erythro- blast differentiation, and early stages of embryonic development, the cells are subject to massive changes in mRNA content and complexity $(17,25,29,37)]$. In addition, for analysis requiring subcellular fractionation, such as characterization of mRNAs encoding secreted proteins (9) or translationally regulated transcripts $(18,25,38)$, obviously neither normalization using housekeeping genes nor total filter hybridization signals may be reliable.

The translation efficiency of a given mRNA species is characterized by its ribosome loading profile [i.e., its distribution between free mRNPs (messenger ribonucleoprotein particles or ribosome-free mRNAs) and polysomebound mRNA pools]. In a given cell, each mRNA species is translated with a characteristic efficiency, which may vary more than 100 -fold for the different cellular mRNAs. Furthermore, translation efficiency of a given mRNA may vary under different cellular conditions (e.g., stimulation, proliferation, differentiation, or drug treatment) (25). Such a regulation is an essential mechanism to control gene expression because it may affect more than $10 \%$ of all expressed mRNA species $(12,25)$.

We have addressed the reliability of array hybridization analyses for the comparison of mRNA populations of different amount and complexities, by comparing for each transcript the signals obtained from the cytoplasmic mRNA population with the signals obtained from the two mutually exclusive ribosome-free (untranslated) and polysome-bound (translated) mRNA populations. Statistical analysis reveals that, for each individual cDNA in the array, the signal intensity from the cytoplasmic mRNA population indeed represents the sum of the signal intensities from the ribosome-free plus the 
polysome-bound samples, after normalization of the values with an exogenous RNA. This is the first formal demonstration that "spiking" of the samples is suitable for quantitative comparisons between RNA populations of different complexities.

\section{MATERIALS AND METHODS}

\section{Cells, RNA Preparation, and Sucrose Gradients}

The murine cytotoxic $\mathrm{T}$ cell clone B6.1.SF.1 (B6.1) $(14,37)$ was cultured in DMEM-10 [DMEM supplemented with $10 \%$ heat-inactivated FCS, $20 \mathrm{mM}$ HEPES, pH 7.0, 0.05 mM $\beta$-mercaptoethanol, $2 \mathrm{mM}$ glutamine, and $1 \%$ X63mIL-2 supernatant as a source of recombinant IL-2 (19)]. Cells in exponential growth were harvested and used either to prepare cytoplasmic mRNA using the NP40 method (28) or for subcellular fractionation in linear $15 \%-40 \%$ sucrose gradients (27).

Aliquots of RNA purified from each fraction were separated through denaturing formaldehyde-agarose gels and transferred to nylon membranes (GeneScreen ${ }^{\circledR}$; NEN ${ }^{\circledR}$ Life Science Products, Boston, MA, USA). rRNA distribution was visualized by methylene blue staining of the filters (26). Northern blots were hybridized with random-primed $[\alpha-32 \mathrm{P}] \mathrm{dCTP}$-labeled cDNA probes. Signals were quantified by phosphorimaging using ImageQuant ${ }^{\mathrm{TM}}$ software (Amersham Pharmacia Biotech, Piscataway, NJ, USA).

\section{cDNA Synthesis, Labeling, Hybridization, and Data Analysis}

Fractions 1-10 (containing free ribonucleoprotein particles) and fractions 11-19 (corresponding to polysomebound mRNA) from the sucrose gradient were pooled. Poly $\left(\mathrm{A}^{+}\right)$mRNAs were purified from these pools and from cytoplasmic RNA, using Oligotex-dT ${ }^{\mathrm{TM}}$ beads (Qiagen, Hilden, Germany) as described (28). Quality and extraction yields were analyzed by filter hybridization after electrophoresis. For subsequent reverse transcription, onefourth of each poly $\left(\mathrm{A}^{+}\right)$mRNA extracted from the ribosome-free and polysome-bound mRNA pools was used, as well as a fraction of poly $\left(\mathrm{A}^{+}\right)$ mRNA from the cytoplasmic RNAs, equivalent to the free plus bound material. Five nanograms of heterologous, polyadenylated exogenous RNA, obtained by in vitro transcription of the plasmid pBluescript ${ }^{\circledR}{ }_{-}$HS59 (a kind gift of J. Paz Ares, CNB-CSIC, Madrid, Spain), containing the cDNA HS59 from Arabidopsis thaliana, were added to each poly $\left(\mathrm{A}^{+}\right)$mRNA sample before cDNA synthesis. Reverse transcription was performed using oligo-dT (Life Technologies, Rockville, MD, USA) as described (24). Reaction products were purified on Sephadex ${ }^{\circledR}$ G-50 spin columns (Roche Molecular Biochemicals, Indianapolis, IN, USA) and the amount of first-strand cDNA quantified by fluorometry (DyNAquant ${ }^{\mathrm{TM}} 200$; Amersham Pharmacia Biotech). Fortyfive nanograms of first-strand cDNA were used to generate $[\alpha-33 \mathrm{P}] \mathrm{dATP}-1 \mathrm{a}-$ beled second-strand cDNA. These radiolabeled targets were used to hybridize four Atlas mouse cDNA arrays (consecutive serial numbers; Clontech Laboratories, Palo Alto, CA, USA), essentially as described (26), using $1 \mu \mathrm{g} / \mathrm{mL}$ mouse $\mathrm{C}_{\mathrm{o}} \mathrm{t}-1$ DNA as competitor. A small filter containing an excess (200 ng) of pBluescript-HS59 plasmid DNA was simultaneously hybridized with each array. Signals were quantified by phosphorimaging using ImageQuant software. Since each cDNA was present in duplicate on the filters, the mean value was calculated. cDNA for which the hybridization values did not verify for the three filters that: $0.7 \times$ mean $_{x, y}<x, y$ $<1.3 \times$ mean $_{x, y}$, were excluded from further analysis (25 cDNAs in all). Linear regression analysis and correlation coefficients were calculated using $\mathrm{Mi}$ crosoft $^{\circledR}$ Excel $^{\circledR} 98$.

\section{RESULTS AND DISCUSSION}

\section{Subcellular RNA Fractionation and Characterization}

RNAs from cytoplasmic extracts were fractionated by sedimentation velocity in $15 \%-40 \%$ sucrose gradients. In these gradients, migration of ribosome-bound transcripts is directly proportional to their ribosome loading, while free mRNAs (in the form of mRNPs) migrate at the top of the gradient (23). The characteristic profile of rRNA distribution in sucrose gradients can be visualized by methylene blue staining of the northern blot (Figure 1A), where the migration of the $40 \mathrm{~S}$ small ribosomal subunit (peak of $18 \mathrm{~S}$ rRNA in fractions 5 and 6) and the migration of the 60S big ribosomal subunit (peak of 28S rRNA in fractions 8 and 9) are visualized. Consequently, it appears that mRNA molecules migrating in fractions 1-9 are not engaged in translation, whereas mRNAs migrating in more dense fractions are loaded with one or more ribosomes and, thus, are being actively translated.

The translational efficiency for each mRNA can be determined by its distribution pattern in sucrose gradients. Indeed, eEF $1 \alpha$ mRNA, coding for the translation elongation factor $1 \alpha$, shows the typical profile for an inefficiently translated transcripts because most of this mRNA is found in the ribosomefree fractions (Figure 1, B and C). Conversely, $\beta$-actin mRNA exhibits a profile characteristic of an efficiently translated transcripts; it is mostly bound to polysomes (Figure 1, B and C). The ratio ribosome-free:polysomebound is an indication of the translation efficiency of the transcript. Here, this ratio was 3.42 and 0.08 for eEF1 $\alpha$ and $\beta$-actin mRNAs, respectively (Figure 1). Several hybridization experiments using cDNA arrays have demonstrated that the ribosome-free:polysome-bound ratios usually range from 10 to 0.08 (25) (additional information at http://www.cnb.uam.es/ jasanz/pradet 2000a), indicating that each mRNA species has a specific, independent distribution between these two mutually exclusive pools.

\section{cDNA Array Hybridization and Analysis}

Array hybridization experiments using cytoplasmic and fractionated ribosome-free and polysome-bound mRNA populations were then performed. Following sucrose gradient fractionation, RNAs from fractions 1-9 (ribosomefree) and from fractions 10-19 (polysome-bound) were pooled. Fraction 20 is routinely discarded because, in addi- 
tion to polysomal mRNAs, it also contains mRNAs attached to high-density cell debris migrating at the bottom of the gradient. Poly $\left(\mathrm{A}^{+}\right)$mRNAs purified from the three RNA populations (ribosome-free, polysome-bound, and cytoplasmic) were used to generate targets for the hybridization of three identical mouse cDNA arrays (Clontech Laboratories) (Figure 2A). After signal quantification, cDNAs whose duplicate hybridization signals deviated more than 1.3-fold from the mean value in all three filters were discarded. This excluded 25 out of the 588 cDNAs in the arrays. The dynamic range of signal intensity for the remaining $566 \mathrm{cDNAs}$ was similar for the three mRNA populations, spanning more than three orders of magnitude (Figure 3 ).

So far, reliability of cDNA array analysis has only been addressed by repeated hybridization of the arrays with the same targets or with targets derived from the same mRNA population, thus demonstrating the reproducibility of the system $(2,32)$. To address the question of reliability in the comparison of divergent mRNAs, we took advantage of the following facts: ( $i)$ the three RNA populations used here have intrinsically different amounts and complexities; (ii) the ribosome-free and polysome-bound RNA populations are mutually exclusive and represent truly independent mRNA populations because each mRNA species has a distinct, characteristic distribution between the two pools; (iii) for each mRNA analyzed, the signal intensity obtained from hybridization with the cytoplasmic targets should be equal to the sum of the signal intensities obtained after hybridization with the ribosome-free and polysome-bound targets. This relation becomes, after a logarithmic transformation (to facilitate graphical representation of the data):

$\log _{10}($ cytoplasmic mRNA $)=\log _{10}$ (ribosome-free mRNA + polysomebound mRNA)

where "mRNA" stands for the abundance of any individual transcript in the indicated pool.

Regression analysis of the data obtained after hybridization of these sam ples showed a significant correlation coefficient $\left(\mathrm{r}^{2}=0.972\right)$ (Figure $\left.2 \mathrm{~B}\right)$ to a line defined by the following equation:

$$
y=0.986 x+0.728
$$

This regression line, although it has a slope close to 1 , does not intersect the coordinate origin, demonstrating that the signals obtained with the cytoplasmic mRNA samples were only proportional to the sum of the ribosome-free plus polysome-bound signals. In other words, for each cDNA, the hybridization signals obtained with each of the three mRNA populations cannot be directly compared. Thus, the variations between the hybridization signals would not be representative of the genuine variations in mRNA expression level in the experiment.

\section{Normalization of Hybridization Data}

To improve data analysis, normalization was assessed by spiking the initial samples with an heterologous poly $(\mathrm{A})^{+}$ tailed transcript generated in vitro from an $A$. thaliana cDNA. The hybridization signal corresponding to this exogenous RNA allowed us to calculate a specific correction factor for each array $\left(K_{C}\right.$ for hybridization with the target corresponding to the cytoplasmic RNAs, $K_{F}$ the ribosome-free, and $K_{B}$ the polysomebound) (Figure 2C). After correction of the hybridization signals, the analysis (Figure 2D) yielded the same correlation coefficient $\left(r^{2}=0.972\right)$ of the data to a line with the following equation:

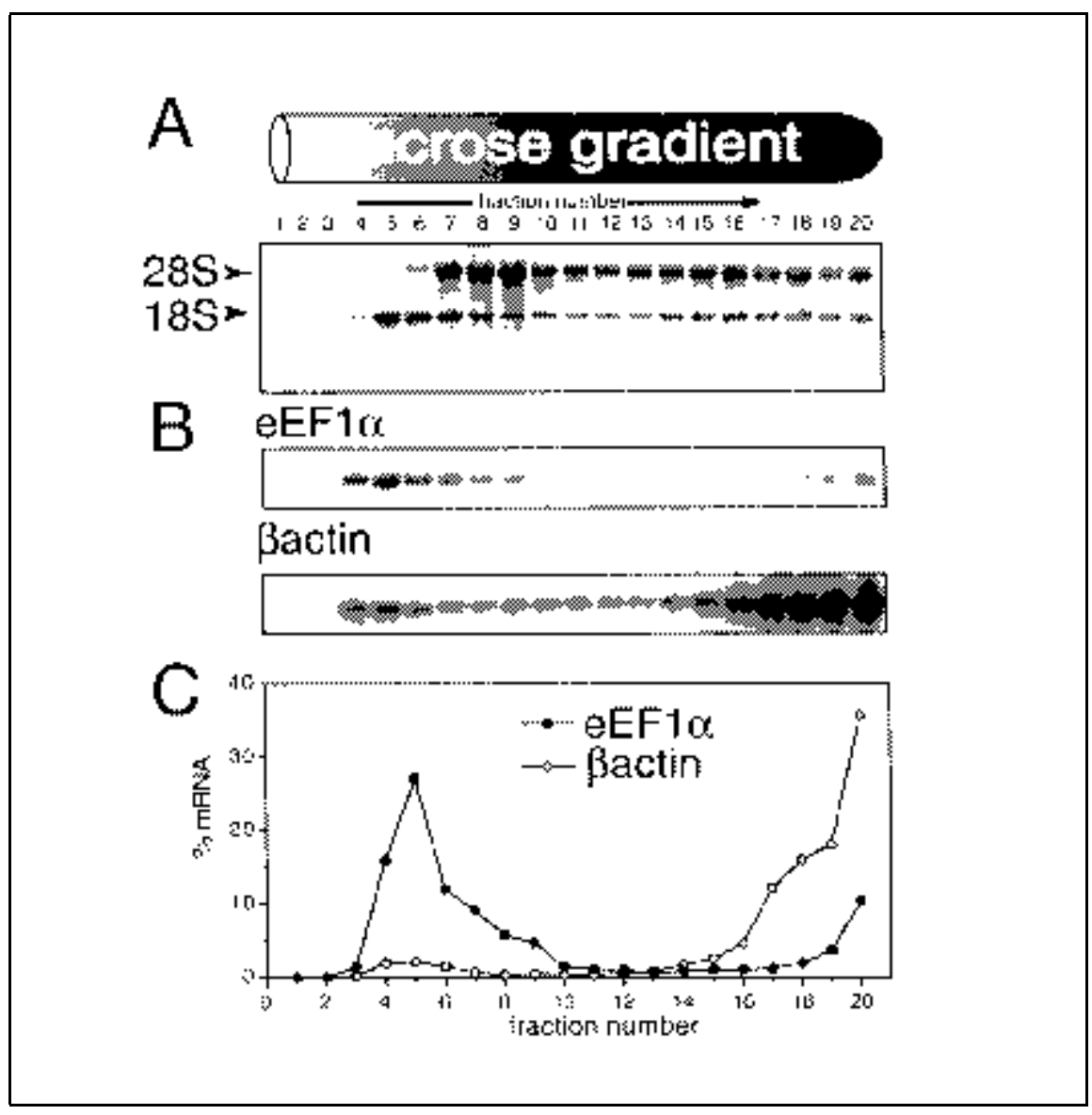

Figure 1. Analysis of RNA fractionation in sucrose gradients. Cytoplasmic extracts from B6.1 cells in exponential growth were layered on top of a 15\%-40\% linear sucrose gradient. After centrifugation, 20 fractions were obtained from the sample, and RNA was purified from each of them. (A) Methylene blue staining of the filter after electrophoretic separation of RNA from each fraction and transfer to a nylon membrane. The migration of $28 \mathrm{~S}, 18 \mathrm{~S}$, and $5 \mathrm{~S}$ rRNAs is indicated. Fraction 1 corresponds to the top, fraction 20 to the bottom. (B) Hybridization signals for eEF1 $\alpha$ and $\beta$-actin mRNAs show typical distributions for either inefficiently (eEF1 $\alpha$ ) or efficiently ( $\beta$-actin) translated mRNAs. (C) Distribution of eEF1 $\alpha$ and $\beta$-actin mRNAs across the sucrose gradient. Data from (B) were quantified by phosphorimaging and plotted as percentage of the total signal, to allow direct comparison between the profiles of two genes, expressed at different levels. 


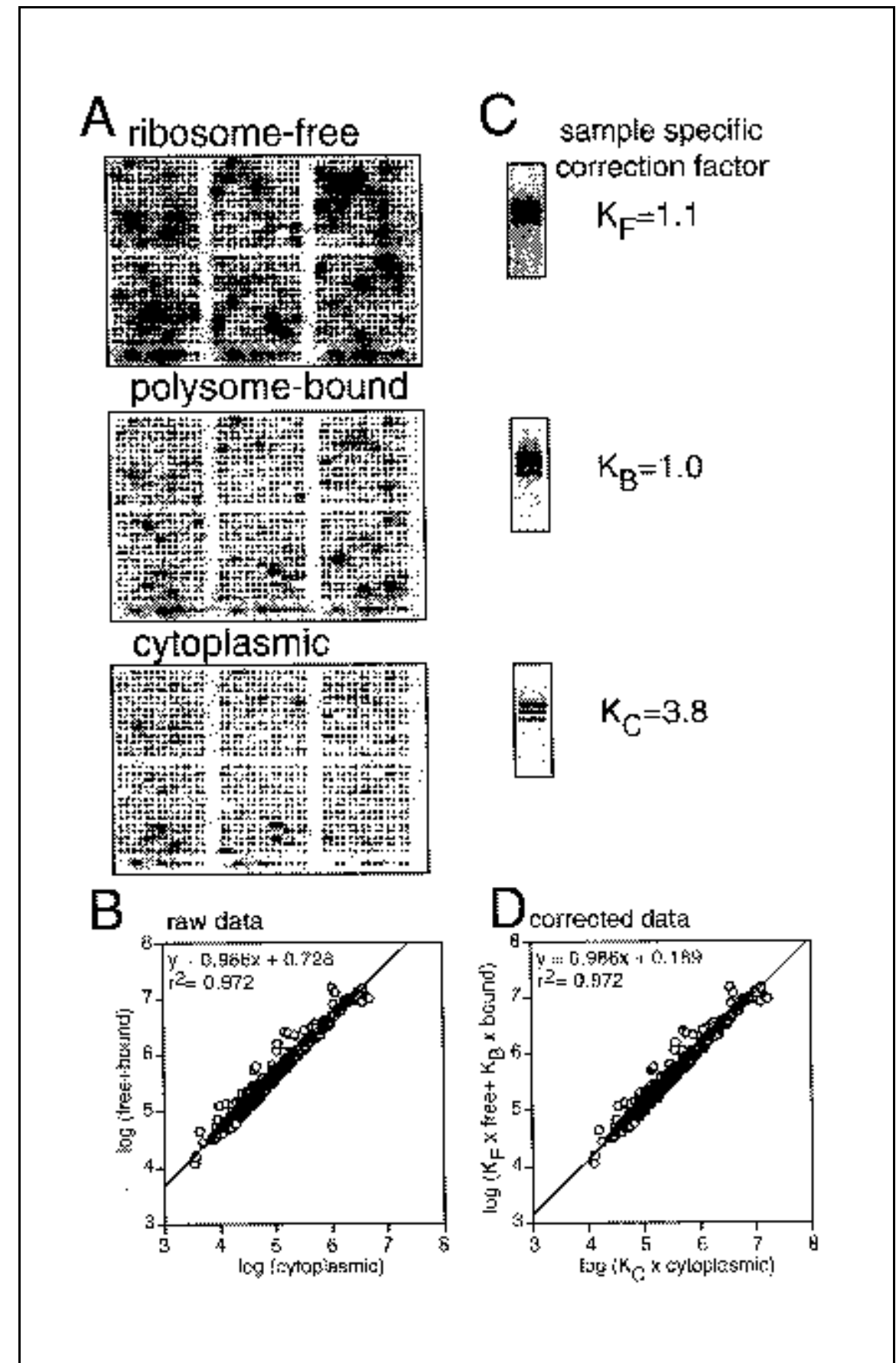

Figure 2. cDNA array hybridization with ribosome-free, polysome-bound, and cytoplasmic B6.1 RNA. mRNA samples were prepared from the ribosome-free pool (fractions 1-9), polysome-bound (fractions 10-19), or cytoplasmic RNA from B6.1 cells. After poly(A) ${ }^{+}$purification, 5 ng exogenous RNA obtained by in vitro transcription of the cDNA HS59 from A. thaliana were added to each sample for normalization purposes. Each of these mixtures was used to prepare a complex cDNA target, which was then hybridized to a cDNA array (Clontech Laboratories), as described in Materials and Methods. (A) Signals obtained after hybridization of cDNA arrays with the ribosome-free, polysome-bound, and cytoplasmic targets (PhosphorImager ${ }^{\circledR}$ scale 0.07-5000; Amersham Pharmacia Biotech). (B) Scatter plot for each of the 563 genes analyzed, representing the signal with the cytoplasmic target versus the sum of the signals from free plus bound targets, after logarithmic transformation of the data values. (C). Hybridization signals for each target with a plasmid containing the $A$. thaliana HS59 cDNA (several bands represent the different mobility forms of the plasmid). The correction factor for each sample was calculated by dividing the exogenous RNA-specific signal from the polysome-bound sample (the highest signal) by the exogenous RNA-specific signal for each target used. (D) Scatter plot representing the signal from the corrected cytoplasmic target versus the sum of the corrected signals from free plus bound targets after correction. 


$$
\mathrm{y}=0.986 \mathrm{x}+0.189
$$

The regression line has still a slope close to one, and this time it intersects the coordinates very close to the origin. This means that, for each cDNA analyzed, the hybridization signals are consistent with the intuitive equation that relates the three mRNA populations:

cytoplasmic mRNA = ribosomefree mRNA + polysome-bound mRNA

Therefore, normalization by spiking of the sample allows a quantitative analysis of mRNA profiling data independently of the amount and complexity of the mRNA in the populations analyzed and allows direct comparison of the data.

Different complexities and changes in RNA abundance may be due to subcellular RNA fractionation but also be the result of physiological processes. Indeed, massive transcription bursts take place, for example, following macrophage and lymphocyte activation $(4,6)$ and during early development
(37). Conversely, generalized transcriptional repression takes place during terminal differentiation processes such as terminal erythropoiesis (29). For these examples, it is crucial that the RNA populations compared reflect the initial cellular conditions. Especially since, because of technical requirements, the same amounts of material are often used for some of the reaction steps, which inevitably results in misrepresentation of the initial sample complexity and amount. Our data demonstrate, for the first time, that spiking of the RNA populations allows one to compensate for this distortion.

Obviously, the exogenous RNA should not hybridize with either the sample RNA to be analyzed or the cDNA array. For this purpose, an $A$. thaliana RNA, which does not hybridize with either murine (data presented here) or human (BP-B and JAG-S; data not shown) cDNA arrays, was used. Furthermore, spiking might also be used for comparisons of samples that cannot be simultaneously hybridized and exposed because of technical limitations. The use of such exogenous RNA for data normalization is readily feasible at low cost, both in terms of time and reagents required. In contrast, all other steps in expression profiling, from RNA extraction to array data analysis, are rather expensive and time consuming.

In conclusion, mRNA profiling emerges as a powerful tool for simultaneous analysis of mRNA expression levels for hundreds to thousands of genes (11). Crucial to data analysis is the issue of accurate normalization, particularly for comparison between divergent samples, which might generate RNA populations with different amounts and complexities. Here, we show that the accurate use of an exogenous RNA for spiking of the samples allows one not only to ensure reproducibility for target synthesis and hybridization but also to restore the initial variations between sample complexities and amounts.

\section{ACKNOWLEDGMENTS}

We are grateful to Dr. M. Hahne for critically reviewing the manuscript, Drs. J. Paz Ares (CNB-CSIC) and M. Nabholz (ISREC, Lausanne) for kindly providing the pBluescript-HS59 plasmid and the B6.1 cell line, respectively, and Ms. C. Mark for editorial help. J.A.G.S. would especially like to thank Dr. Carlos Martinez-A. for continuous support and encouragement. This research was partially supported by an EU TMR Network grant (contract no. ERBFMRXCT980197) (to J.A.G.S. and E.W.M.) and by the Austrian National Bank, and the Austrian Fonds zur Foerderung der Wissenschaftlichen Forschung, FWF (E.W.M.). The Department of Immunology and Oncology was founded and is supported by the Spanish Research Council (CSIC) and Pharmacia Corporation.

\section{REFERENCES}

1.Adryan, B., V. Carlguth, and H.J. Decker. 1999. Digital image processing for rapid analysis of differentially expressed transcripts on high-density cDNA arrays. BioTechniques 26:1174-1179.

2.Bernard, K., N. Auphan, S. Granjeaud, G.
Figure 3. Dynamic range of cDNA array hybridization signals using different targets. Specific hybridization signals for each filter were sorted by increasing values and plotted against a running index in the $\mathrm{x}$-axis. The dynamic range of the signal intensities after hybridization with cytoplasmic, ribosomefree, or polysome-bound targets spans over four orders of magnitude in all cases. 
Victorero, A.M. Schmitt-Verhulst, B.R. Jordan, and C. Nguyen. 1996. Multiplex messenger assay: simultaneous, quantitative measurement of expression of many genes in the context of T cell activation. Nucleic Acids Res. 24:1435-1442.

3.Brown, P.O. and D. Botstein. 1999. Exploring the new world of the genome with DNA microarrays. Nat. Genet. 21:33-37.

4.Celada, A. and C. Nathan. 1994. Macrophage activation revisited. Immunol. Today 15:100-102.

5.Chen, Y., E.R. Dougherty, and M.L. Bittner. 1997. Ratio-based decissions and the quantitative analysis of cDNA microarray images. J. Biomed. Optics 2:364-374.

6.Crabtree, G.R. 1989. Contingent genetic regulatory events in $\mathrm{T}$ lymphocyte activation. Science 243:355-361.

7.DeRisi, J., L. Penland, P.O. Brown, M.L. Bittner, P.S. Meltzer, M. Ray, Y. Chen, Y.A. Su, and J.M. Trent. 1996. Use of a cDNA microarray to analyse gene expression patterns in human cancer. Nat. Genet. 14:457460.

8.DeRisi, J.L., V.R. Iyer, and P.O. Brown. 1997. Exploring the metabolic and genetic control of gene expression on a genomic scale. Science 278:680-686.

9.Diehn, M., M.B. Eisen, D. Botstein, and P.O. Brown. 2000. Large-scale identification of secreted and membrane-associated gene products using DNA microarrays. Nat. Genet. 25:58-62.

10.Eisen, M.B. and P.O. Brown. 1999. DNA arrays for analysis of gene expression. Methods Enzymol. 303:179-205.

11.Eisen, M.B., P.T. Spellman, P.O. Brown, and D. Botstein. 1998. Cluster analysis and display of genome-wide expression patterns. Proc. Natl. Acad. Sci USA 95:14863-14868.

12.Garcia-Sanz, J.A., W. Mikulits, A. Livingstone, I. Lefkovits, and E.W. Müllner. 1998. Translation control: a general mechanism for gene regulation during $\mathrm{T}$ cell activation. FASEB J. 12:299-306.

13.Gerhold, D., T. Rushmore, and C.T. Caskey. 1999. DNA chips: promising toys have become powerful tools. Trends Biochem. Sci. 24:168-173.

14.Gillis, S., M.M. Ferm, W. Ou, and K.A. Smith. 1978. T cell growth factor: parameters of production and a quantitative microassay for activity. J. Immunol. 120:2027-2032.

15.Huang, F., J. Adelman, H. Jiang, N.I. Goldstein, and P.B. Fisher. 1999. Identification and temporal expression pattern of genes modulated during irreversible growth arrest and terminal differentiation in human melanoma cells. Oncogene 18:3546-3552.

16.Iyer, V.R., M.B. Eisen, D.T. Ross, G. Schuler, T. Moore, J.C.F. Lee, J.M. Trent, L.M. Staudt et al. 1999. The transcriptional program in the response of human fibroblasts to serum. Science 283:83-87.

17.Jedlicka, P. and R. Panniers. 1991. Mechanism of activation of protein synthesis initiation in mitogen- stimulated T lymphocytes. J. Biol. Chem. 266:15663-15669.

18.Johannes, G., M.S. Carter, M.B. Eisen, P.O. Brown, and P. Sarnow. 1999. Identification of eukaryotic mRNAs that are translated at re- duced cap binding complex eIF4F concentrations using a cDNA microarray. Proc. Natl. Acad. Sci. USA 96:13118-13123.

19. Karasuyama, H. and F. Melchers. 1988. Establishment of mouse cell lines which constitutively secrete large quantities of interleukin 2, 3, 4 or 5 using modified cDNA expression verctors. Eur. J. Immunol. 18:98-104.

20.Lipshutz, R.J., S.P. Fodor, T.R. Gingeras, and D.J. Lockhart. 1999. High density synthetic oligonucleotide arrays. Nat. Genet. $21: 20-24$.

21.Lu, P., J.A. Garcia-Sanz, M.G. Lichtenheld, and E.R. Podack. 1992. Perforin expression in human peripheral blood mononuclear cells. Definition of an IL-2-independent pathway of perforin induction in $\mathrm{CD} 8+\mathrm{T}$ cells. $\mathrm{J}$. Im munol. 148:3354-3360.

22.Marton, M.J., J.L. DeRisi, H.A. Bennett, V.R. Iyer, M.R. Meyer, C.J. Roberts, R. Stoughton, J. Burchard et al. 1998. Drug target validation and identification of secondary drug target effects using DNA microarrays. Nat. Med. 4:1293-1301.

23.Mathews, M.B., N. Sonenberg, and J.W.B. Hershey. 1996. Origins and targets of translational control, p. 1-30. In J.W.B. Hershey, M.B. Mathews, and N. Sonenberg (Eds.), Translational Control. CSH Laboratory Press, Cold Spring Harbor, NY

24.Mikulits, W., H. Dolznig, R. Hofbauer, and E.W. Müllner. 1999. Reverse strand priming: a versatile cDNA radiolabeling method for differential hybridization on nucleic acid arrays. BioTechniques 26:846-850

25.Mikulits, W., B. Pradet-Balade, B. Habermann, H. Beug, J.A. Garcia-Sanz, and E.W. Müllner. 2000. Isolation of translationally controlled mRNAs by differential screening. FASEB J. 14:1641-1652.

26.Müllner, E.W., and J.A. Garcia-Sanz. 1997. Analysis of RNA expression by northern blotting, p. 407-424. In I. Lefkovits (Ed.), Im munology Methods Manual, vol. 1. Academic Press, London.

27.Müllner, E.W. and J.A. Garcia-Sanz. 1997. Polysome gradients, p. 457-462. In I. Lefkovits (Ed.), Manual of Immunological Methods, vol. 1. Academic Press, London.

28.Müllner, E.W. and J.A. Garcia-Sanz. 1997. Preparation of RNA, p. 389-406. In I. Lefkovits (Ed.), Manual of Immunological Methods, vol. 1. Academic Press, London.

29.Munro, H.N. 1990. Iron regulation of ferritin gene expression. J. Cell Biochem. 44:107115 .

30.Papayannopoulou, T. and J. Abkowitz. 1991. Biology of erythropoiesis, erythroid differentiation and maturation, p. 242-254. In R. Hoffman, E.J. Benz, S.J. Shatil, B. Furie, and H.J. Cohen (Eds.), Hematology: Basic Principles and Practice. Churchill Livingstone, New York.

31.Perou, C.M., S.S. Jeffrey, M. van de Rijn, C.A. Rees, M.B. Eisen, D.T. Ross, A. Pergamenschikov, C.F. Williams et al. 1999. Distinctive gene expression patterns in human mammary epithelial cells and breast cancers. Proc. Natl. Acad. Sci. USA 96:9212-9217.

32.Pollack, J.R., C.M. Perou, A.A. Alizadeh, M.B. Eisen, A. Pergamenschikov, C.F. Williams, S.S. Jeffrey, D. Botstein, and P.O.
Brown. 1999. Genome-wide analysis of DNA copy-number changes using cDNA microarrays. Nat. Genet. 23:41-46.

33.Schuchhardt, J., D. Beule, A. Malik, E. Wolski, H. Eickhoff, H. Lehrach, and $H$. Herzel. 2000. Normalization strategies for cDNA microarrays. Nucleic Acids Res. 28:e47.

34.Spellman, P.T., G. Sherlock, M.Q. Zhang, V.R. Iyer, K. Anders, M.B. Eisen, P.O. Brown, D. Botstein, and B. Futcher. 1998. Comprehensive identification of cell cycleregulated genes of the yeast Saccharomyces cerevisiae by microarray hybridization. Mol. Biol. Cell 9:3273-3297.

35.Trenkle, T., F. Mathieu-Daude, J. Welsh, and M. McClelland. 1999. Reduced com plexity probes for DNA arrays. Methods Enzymol. 303:380-392.

36.Trenkle, T., J. Welsh, B. Jung, F. MathieuDaude, and M. McClelland. 1998. Non-stoichiometric reduced complexity probes for cDNA arrays. Nucleic Acids Res. 26:38833891.

37.von Boehmer, H., H. Hengartner, M. Nabholz, W. Lernhardt, M.H. Schreier, and W. Haas. 1979. Fine specificity of a continuously growing killer cell clone specific for $\mathrm{H}-\mathrm{Y}$ antigen. Eur. J. Immunol. 9:592-597.

38. Wickens, M., J. Kimble, and S. Strickland. 1996. Translational control of developmental decisions, p. 411-450. In J.W.B. Hershey, M.B. Mathews, and N. Sonenberg (Eds.), Translational Control. CSH Laboratory Press, Cold Spring Harbor, NY

39.Zong, Q., M. Schummer, L. Hood, and D.R. Morris. 1999. Messenger RNA translation state: the second dimension of high- throughput expression screening. Proc. Natl. Acad. Sci. USA 96:10632-10636.

Received 18 October 2000; accepted 18 December 2000.

Address correspondence to:

Dr. Jose A. Garcia-Sanz

Department of Immunology and Oncology Centro Nacional de Biotecnologia (CNB-CSIC) Campus de Cantoblanco

E-28049, Madrid, Spain

e-mail: jasanz@cnb.uam.es 